\title{
Self-Assembled Faceted Mesocrystals: Advances in Optimization of Growth Conditions
}

Julian Johannes Schlotheuber née Brunner ${ }^{1}$, Britta Maier ${ }^{1}$, Felizitas Kirner ${ }^{1}$, Sebastian Sturm², Helmut Cölfen ${ }^{l} *$ and Elena V. Sturm ${ }^{l} *$

${ }^{1}$ University of Konstanz, Physical Chemistry, Universitätsstraße 10, PF714, 78457 Konstanz

${ }^{2}$ Leibniz Institute for Solid State and Materials Research (IFW) Dresden, Helmholtzstraße 20, 01069, Dresden, Germany

*Email: elena.sturm@uni-konstanz.de, helmut.coelfen@uni-konstanz.de

Mesocrystal formation, nonclassical crystallization, nanoparticle, magnetite 

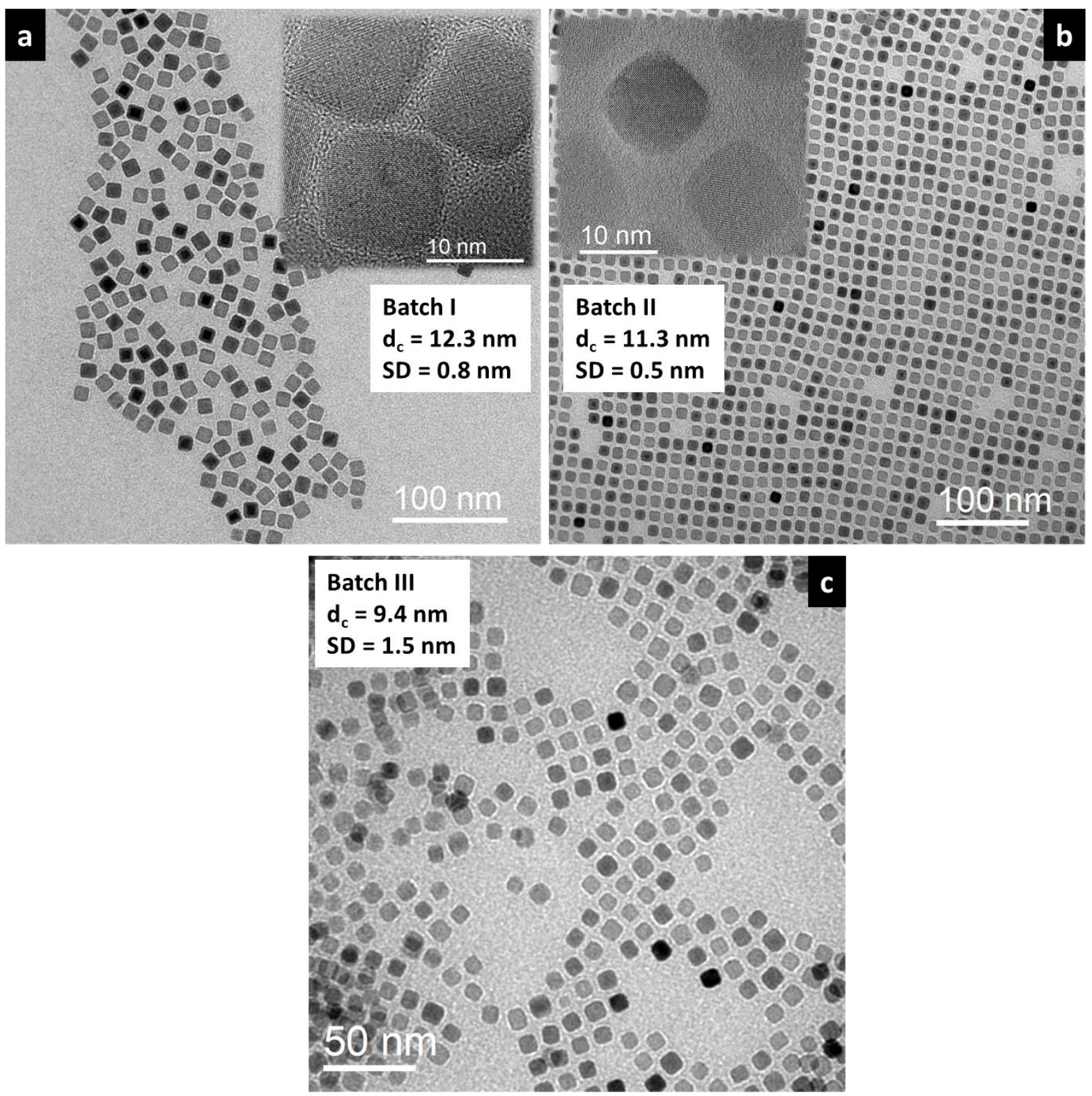

Figure S1. (a, b, c) TEM images of nanocubes from batches I, II and III and their corresponding core diameters $\left(\mathrm{d}_{\mathrm{c}}\right.$, i.e., edge length of idealized cube) and standard deviations (SD). Insets in (a) and (b) show the HRTEM images of nanocubes. 

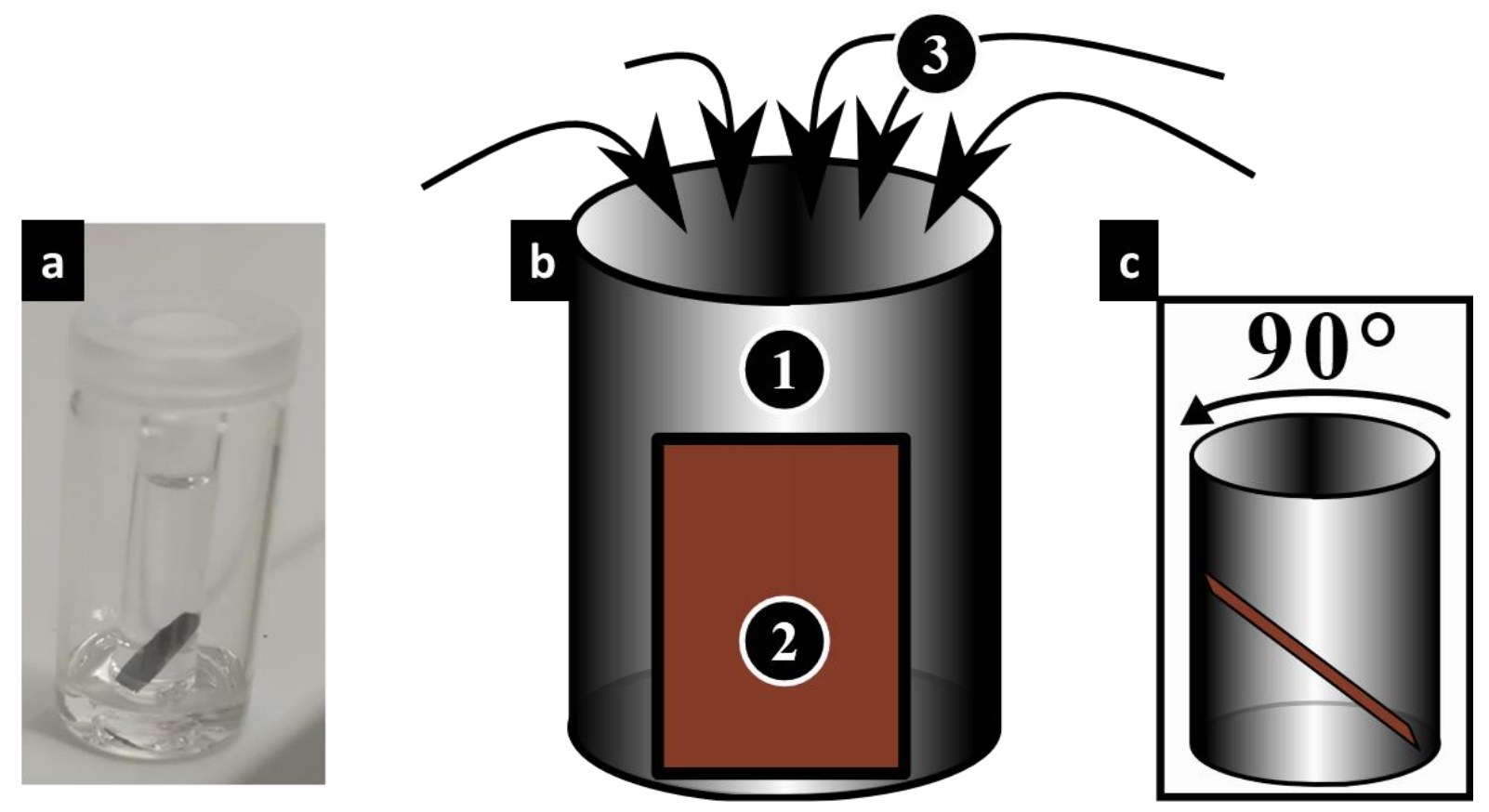

Figure S2. Experimental setup for the growth of mesocrystals by means of the gas phase diffusion technique. (a) Photograph of the setup. (b, c) Schematic illustration of (a): a glass tube containing nanoparticle dispersion (1) and a silicon snippet (2) (positioned at vertically inclined orientation) is stored in a sealed glass vial containing the destabilizing diffusion phase (i.e., nonsolvent or poor solvent) (3). Scheme in (c) shows the side view of a glass vial. 

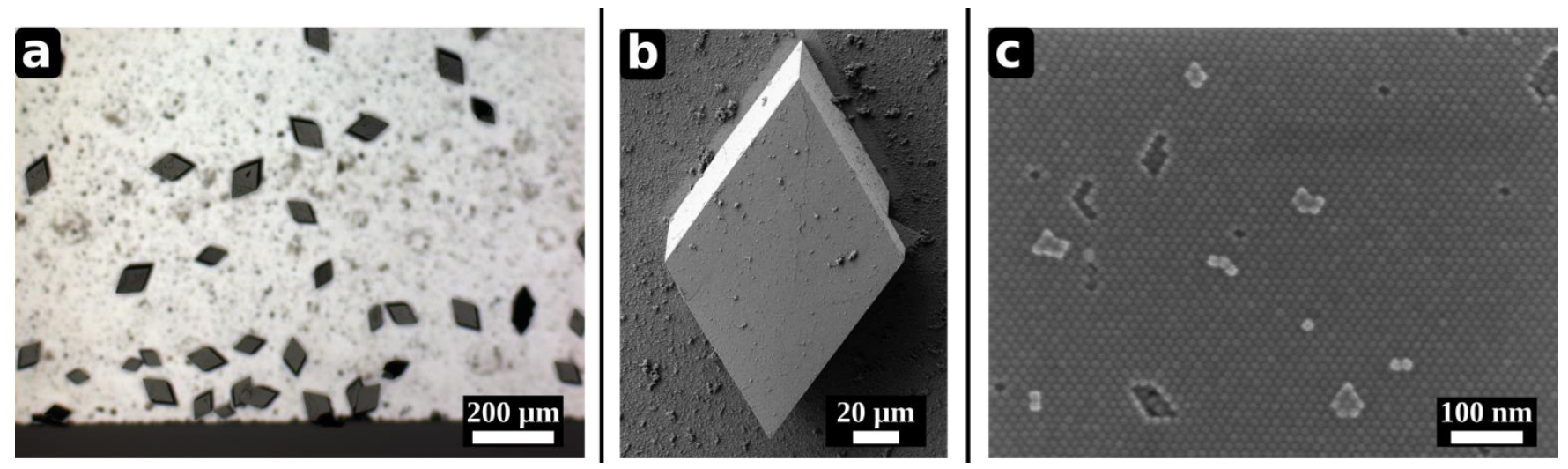

Figure S3. Different image magnification of a faceted mesocrystal (batch I, cyclohexane, ethanol). (a) Light microscope image (b) Scanning electron microscope image of a faced mesocrystal (c) Scanning electron image of a faceted mesocrystal surface. Further images and analytics of such mesocrystals can be found in the following literature. ${ }^{1-3}$
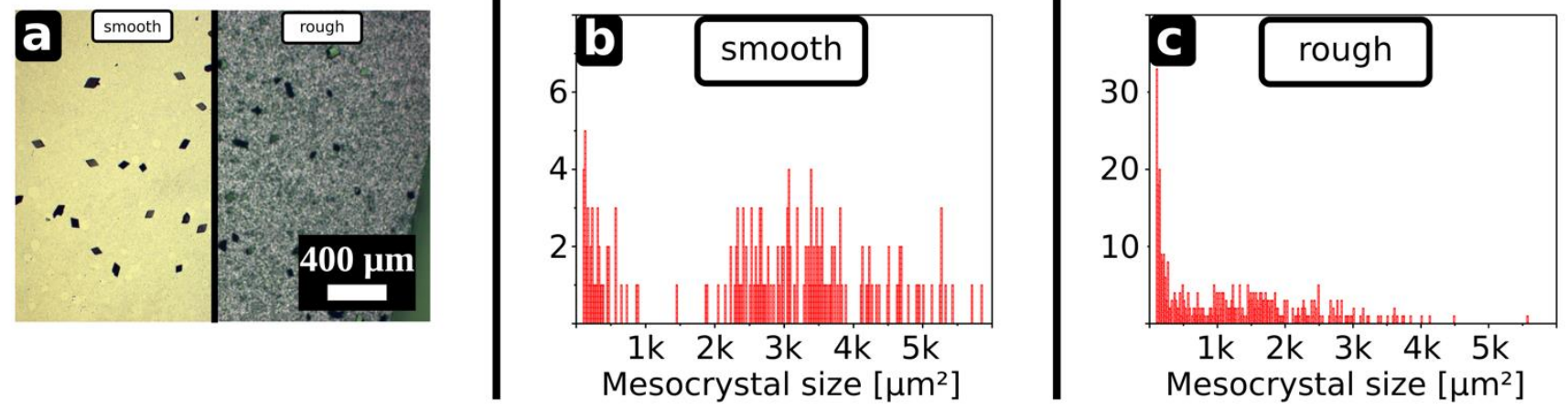

Figure S4. Comparison of mesocrystals grown on a smooth or a rough silicon snippet respectively under otherwise similar conditions (batch I heptane, ethanol). Only mesocrystals with areas between 100 to $10000 \mu \mathrm{m}^{2}$ were counted. (a) Light microscope images of grown mesocrystals on a rough and a smooth silicon snippet. (b) Histogram of counted mesocrystal areas from a polished silicon snippet. The mean mesocrystal area is $2800 \mu \mathrm{m}^{2}$, count $=181$. c) Histogram of mesocrystal areas from the rough side of an equivalent silicon snippet. The mean mesocrystal area is $1500 \mu \mathrm{m}^{2}$, count $=405$. 


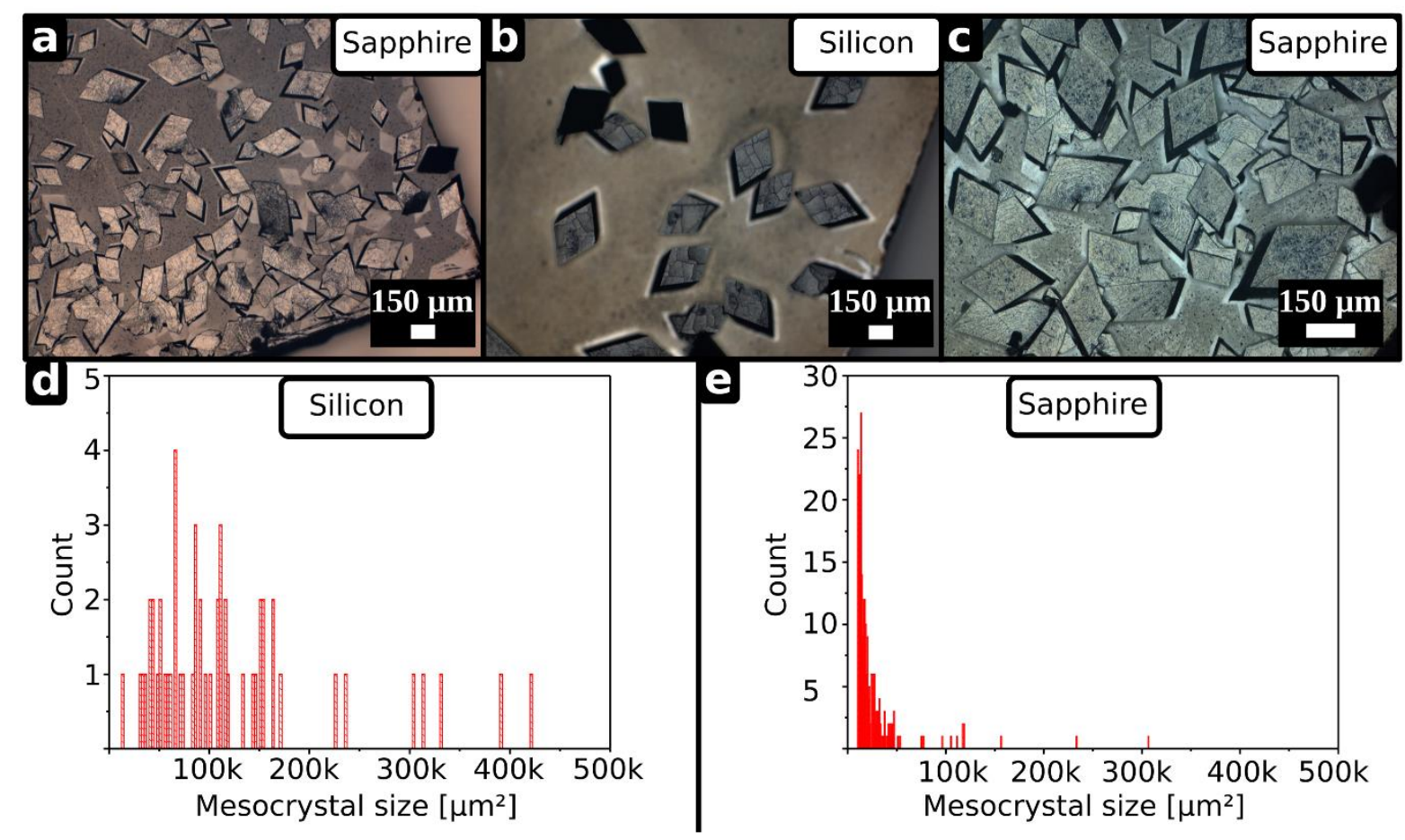

Figure S5. Formed mesocrystals on different substrates (polished silicon and sapphire) under the same conditions (batch I, cyclohexane, 1-propanol). Mesocrystals were only counted with areas between 10000 to $700000 \mu \mathrm{m}^{2}$. (a-c) Light microscope images of the formed mesocrystals. (a,b) have the same initial nanocrystal concentration $(11 \mathrm{mg} / \mathrm{mL})$, while (c) starts with a higher initial concentration (12 mg/mL). (d) A histogram of single mesocrystal areas from growth on a polished silicon snippet is shown (mesocrystal area $121000 \mu \mathrm{m}^{2}$, count $=53$ ). (e) A histogram of mesocrystal areas from growth on a polished sapphire snippet is shown (mesocrystal area 28000 $\mu \mathrm{m}^{2}$, count $=248$ ). 


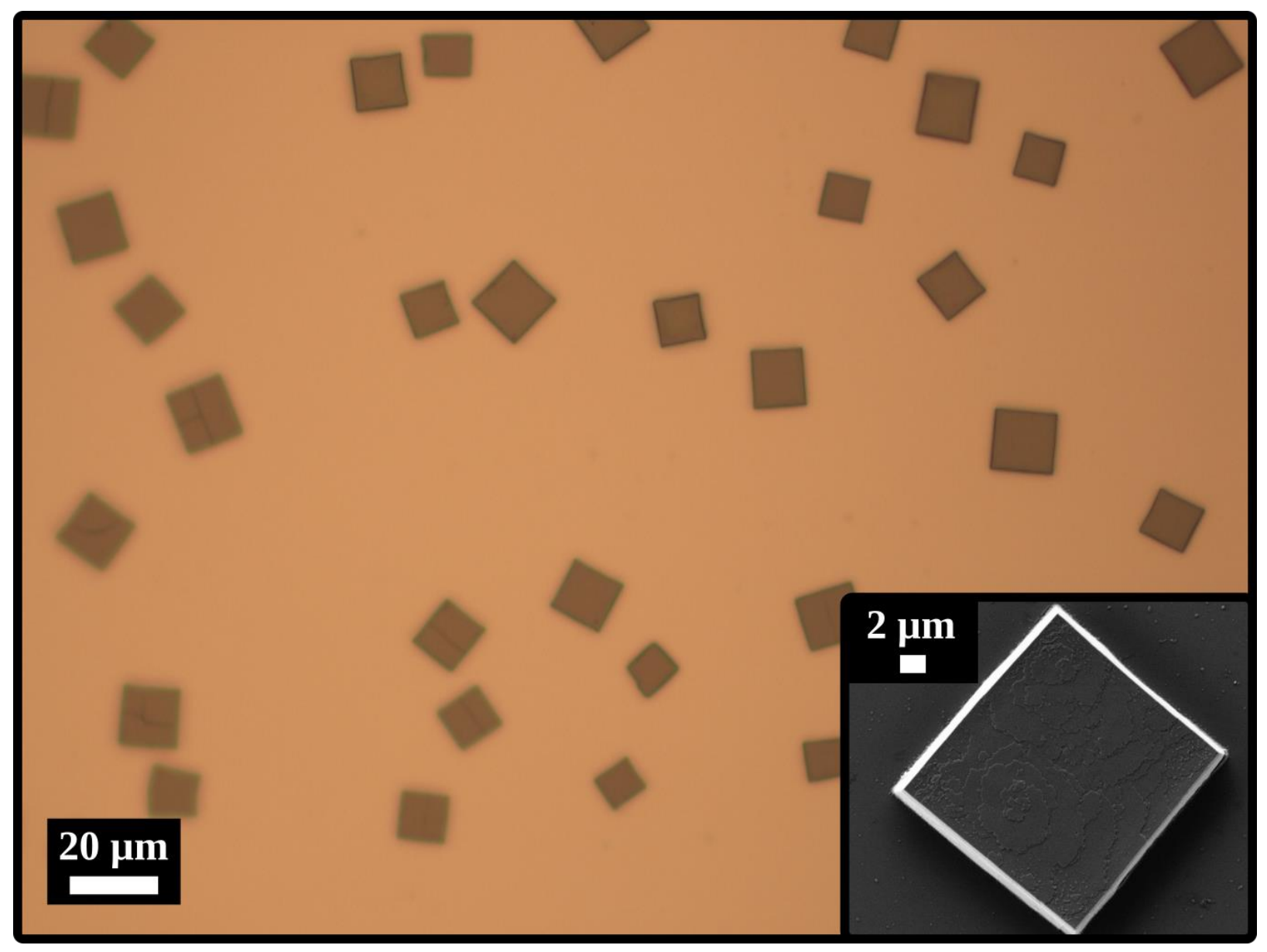

Figure S6. Formed mesocrystals from nanocrystal batch II with oleic acid as an additive (THF, ethanol). 




Figure S7. Optimizing additive concentration. Investigations with a light microscope on the influence of different concentrations of excess additive concentration (batch III, THF, ethanol). The nanocrystal concentration was $3 \mathrm{mg} / \mathrm{mL}$. (a) No mesocrystals formed. (b) Mesocrystals form for low concentration of excess oleic acid. The overall mesocrystal morphology is mainly rhombohedral. c-e) Tetragonal prisms become predominant for additive concentrations above 5 $\mu \mathrm{L} / \mathrm{mL}$. With increasing oleic acid concentration, an oily film appears leading to charging effects for the analysis using SEM. 


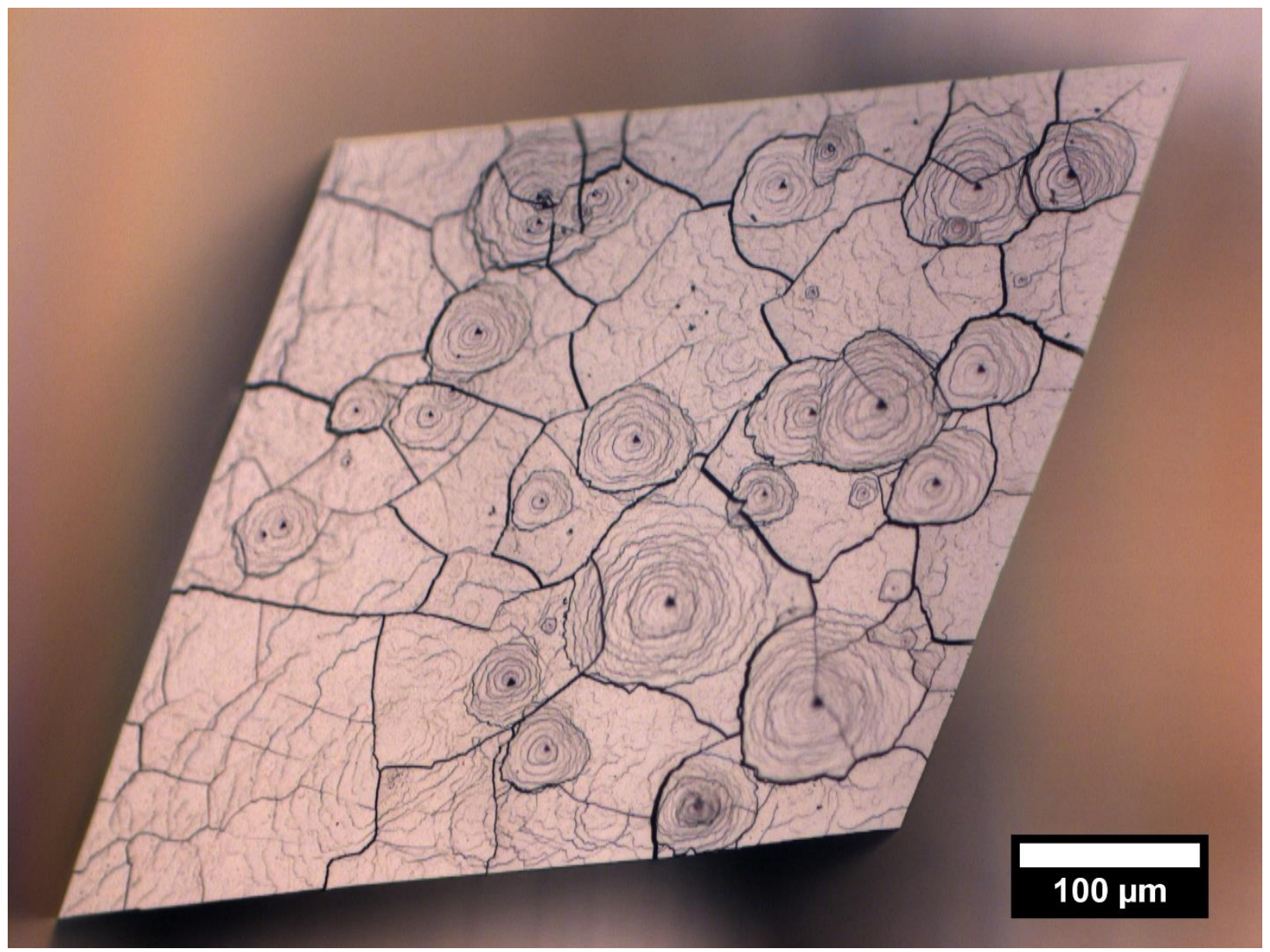

Figure S8. A large mesocrystal grown from nanocrystal batch I in heptane. The destabilizing agent was n-propanol/heptane (1:1). The initial nanocrystal concentration is $15 \mathrm{mg} / \mathrm{mL}$. 


\section{REFERENCES}

(1) Brunner, J.; Cölfen, H. Progress in Mesocrystal Formation. No Crystallization via Nonclassical Pathways Volume 1: Nucleation, Assembly, Observation \& Application, Part 4 - Progress in Mesocrystal Formation; 2020; lpp 73-96.

(2) Opel, J.; Brunner, J.; Zimmermanns, R.; Steegmans, T.; Sturm, E.; Kellermeier, M.; Cölfen, H.; García-Ruiz, J. M. Symbiosis of Silica Biomorphs and Magnetite Mesocrystals. Adv. Funct. Mater. 2019, 29 (37), 1902047.

(3) Brunner, J.; Maier, B.; Rosenberg, R.; Sturm, S.; Cölfen, H.; Sturm, E. V. Nonclassical Recrystallization. Chem. - A Eur. J. 2020, 26 (66), 15242-15248. 